\title{
Evaluación de la trayectoria de la restauración en un bosque andino a través de redes mutualistas de dispersión de semillas
}

\section{Evaluation of the restoration trajectory of an Andean forest through seed dispersal mutualistic networks}

\author{
Astrid Ramírez ${ }^{1 *(1)}$ y Ángela Parrado-Rosselli ${ }^{\circledR}$
}

Ramírez, A. y Parrado-Rosselli, A. (2021). Evaluación de la trayectoria de la restauración en un bosque andino a través de redes mutualistas de dispersión de semillas. Colombia Forestal, 24(1), 108-122

Recepción: 28 de noviembre 2019

\section{Resumen}

Con el fin de evaluar la restauración desde un enfoque funcional se compararon las interacciones de dispersión de semillas por aves entre un bosque restaurado y el de referencia. Se registró la abundancia y riqueza de aves frugívoras y plantas ornitócoras; del mismo modo, se generaron matrices de interacción con base en el consumo de frutos y el solapamiento morfológico entre el tamaño del fruto y la comisura de las aves. Los resultados mostraron que el número de enlaces por especie y anidamiento fueron mayores en el bosque de referencia; mientras que en el restaurado se presentó mayor variación mensual del número de interacciones. Además, la especie más conectada en los dos bosques fue Diglossa cyanea. Se discute que, aunque se restablecieron algunas interacciones, aún hay diferencias entre bosques y por ello se debe hacer gestión forestal como enriquecimiento con plantas ornitócoras que permita aumentar las interacciones y el banco de semillas.

Palabras clave: bosques andinos, bosque de referencia, métricas de red, parque ecológico La Poma.
Aprobación: 21 de septiembre 2020

\begin{abstract}
In order to evaluate the restoration from a functional approach, we compared avian seed dispersal interactions between a restored forest and a reference site. For this purpose, abundance and species richness of frugivorous birds and ornitochours plants were recorded. Then, interaction matrices were generated based on fruit consumption and the morphological overlap between fruit and bill size. Our results show that the number of links per species and nestedness, were higher in the reference forest, while the number of interactions varied greatly in the restored forest. Furthermore, the most connected species in the two forests was the Masked Flowerpierce (Diglossa cyanea). We discussed that although some interactions were reestablished, there are still some differences between sites and hence, management actions such as enrichment with ornithochorous plants, in order to enhance interactions and to improve the seed bank should be promoted.
\end{abstract}

Keywords: andean forest, reference forest, seed dispersal, network metrics, La Poma ecological park.

Universidad Distrital Francisco José de Caldas. Bogotá, Colombia. avramirezc@correo.udistrital.edu.co. Autora para correspondencia 


\section{INTRODUCCIÓN}

Uno de los objetivos de la restauración ecológica es recuperar la composición, estructura y función de los ecosistemas. Por tal, el seguimiento continuo es fundamental para lograr tal propósito y evaluar si los objetivos de la restauración se han alcanzado. Por lo general, el monitoreo de la restauración se ha centrado en evaluar y comparar (entre el sitio restaurado con el ecosistema de referencia) aspectos composicionales como la riqueza y la abundancia de especies vegetales (Alanís-Rodríguez et al., 2008; Durigan et al., 2015; Murcia y Guariguata, 2014). Sin embargo, son pocas las aproximaciones que intentan evaluar cómo se han recuperado los aspectos funcionales de los sitios restaurados y solo recientemente se ha hecho énfasis en la necesidad de monitorear dichas características (Chazdon et al., 2016; Durigan et al., 2015).

Las interacciones ecológicas son uno de los aspectos funcionales que se ha sugerido monitorear (Kaiser-Bunbury et al., 2010; Ribeiro da Silva et al., 2015). Por ejemplo, la dispersión de semillas por aves es una interacción mutualista clave en el mantenimiento de los ecosistemas la cual, en el mediano plazo, incide en la composición y estructura de los bosques. Además, la dispersión tiene gran importancia en la aceleración de los procesos de restauración, pues contribuye a enriquecer el banco de semillas en el suelo, a la colonización de nuevos sitios y a la distribución y abundancia de las especies de plantas (Howe y Smallwood, 1982; Wunderle, 1997). En los bosques tropicales, donde más del $70 \%$ de las especies de plantas están adaptadas para la dispersión de sus semillas por animales (Arbeláez y Parrado-Rosselli, 2005; Link y Stevenson, 2004; Yockteng y Cavelier, 1998), el restablecimiento temprano de las relaciones de dispersión entre plantas y animales favorecería la restauración pasiva y, por ende, tendría incidencia en la disminución de costos en los proyectos de restauración.

En los últimos años las interacciones entre plantas y animales, principalmente de dispersión y polinización, se han analizado mediante redes mutualistas. Estas redes permiten entender los efectos de la aparición o desaparición de una determinada especie en el funcionamiento de la red y, por ende, pueden proporcionar indicadores útiles para guiar y evaluar los objetivos de la restauración (Fontaine et al., 2005; García, 2016). Además, las redes mutualistas proveen información acerca de la estabilidad y resiliencia de los ecosistemas (Bascompte, 2009; Tylianakys et al., 2010).

Para la construcción de las redes de mutualistas, además de las observaciones directas sobre las visitas por parte de la fauna a las especies en flor o en fruto, algunos autores han propuesto modelaciones que permitan predecir o prever interacciones entre especies. Por ejemplo, Donoso et al. (2017) y Bender et al. (2018) propusieron la utilización de variables como el tamaño de los elementos que interactúan tales como fruto y el pico de las aves, con lo que se asumiría, por ejemplo, que aves pequeñas no pueden alimentarse ni dispersar frutos/semillas de gran tamaño. Esto implica que habría interacciones prohibidas basadas en los tamaños y las morfologías (Bender et al., 2018; Dehling et al., 2016; Kelly et al., 2010).

De esta forma, cuando los rasgos coinciden se promoverían o darían interacciones potenciales en la red; mientras que cuando los rasgos no coinciden se generarían los Ilamados enlaces prohibidos. Estos últimos corresponden a restricciones en la interacción debido a la falta de complementariedad de rasgos. Otros aspectos que también provocarían restricciones (aceptación o prohibición) de interacción son la abundancia, la fenología, la distribución espacial de las especies y las relaciones filogenéticas (Santamaría y Rodríguez-Gironés, 2007; Stang et al., 2007; Vázquez et al., 2007).

Una de las más recientes aplicaciones de redes mutualistas en la evaluación de proyectos de restauración fue el trabajo realizado por Ribeiro da Silva et al. (2015). En dicho trabajo, desarrollado en tres bosques restaurados de la Mata Atlántica con diferentes años de antigüedad, se comparó la estructura de las redes de dispersión de semillas 
por aves y se encontró que a medida que avanzaba el tiempo desde la restauración aumentaban las interacciones entre especies y la modularidad; lo que significa que las especies tendían a formar grupos de interacción (módulos) que se relacionaban entre sí. De igual manera, Forup et al. (2008) y Williams et al. (2011) encontraron que las redes de interacción de plantas y polinizadores en bosques restaurados fueron significativamente menos complejas y con una menor conectividad en comparación con el bosque de referencia.

A pesar de lo anterior, la mayoría de estos estudios han sido desarrollados en el mediterráneo y en Norte América (p. ej. Kaiser-Bunbury et al., 2010; Nogales et al., 2015; Williams, 2011). En contraste, en ecosistemas tropicales y particularmente en la región Andina, es escasa la información sobre redes mutualistas (Bender et al., 2018; Burgos et al., 2003; Palacio et al., 2016) y menor aún su utilización como herramienta para el monitoreo de la trayectoria de la restauración. En el caso particular de los bosques andinos colombianos los pocos estudios existentes han encontrado que, en situaciones no perturbadas, las redes de dispersión planta-ave son anidadas, asimétricas y heterogéneas (Burgos et al., 2003; Palacio et al., 2016), lo que coincide con diferentes estudios realizados en otras partes del mundo (p. ej. Bascompte, 2009; García, 2016; Kaiser-Bunbury et al., 2010; Nogales et al., 2015; Ribeiro da Silva et al., 2015; Vázquez et al., 2009). Por ende, sería de esperarse que, en bosques restaurados, debido a la ausencia de ciertas especies de plantas y animales que aún no se han logrado establecer o a las diferencias en la dominancia de las especies en relación a su sistema de referencia, las redes de dispersión de semillas sean menos anidadas, simétricas y con un bajo número de interacciones.

En los bosques andinos secos de los alrededores de Bogotá, Colombia, la Corporación Ambiental Empresarial (adscrita a la Cámara de Comercio de Bogotá) ha desarrollado desde 1996 un proceso de restauración en el parque ecológico la Poma. Allí se encontró que, producto de este proceso, se ha recuperado parcialmente la comunidad de aves; pero, de igual modo, que ciertos grupos tróficos, particularmente aves que consumen néctar y frutos, aún son escasos en términos de abundancia y riqueza (Ramírez, 2019). Esto podría repercutir en procesos ecológicos como la polinización y dispersión de semillas y, por ende, interacciones ecológicas clave podrían no haberse recuperado.

Por lo tanto, con el fin de evaluar la trayectoria de la restauración desde una aproximación funcional el objetivo de esta investigación fue comparar la estructura de la red mutualista entre plantas ornitócoras y aves frugívoras en un bosque andino restaurado hace 22 años y el bosque de referencia, esto a partir observaciones, reportes de literatura y rasgos de tamaño. Para tal, se caracterizó la variación mensual de la abundancia y riqueza de la comunidad de aves frugívoras y plantas ornitócoras en fruto. En segundo lugar, se caracterizó el consumo de frutos a partir de observaciones, reportes de literatura y se realizó un modelo de interacción entre tamaño de la comisura, longitud del culmen, altura del pico de las aves y el tamaño del fruto (longitud ecuatorial y longitudinal). En tercer lugar, se construyeron redes cualitativas y cuantitativas por bosque sobre las relaciones potenciales de dispersión de semillas. Teniendo en cuenta la lentitud de la recuperación de los bosques andinos en términos de biodiversidad y funciones ecológicas (Groenendijk, 2005), se esperaría que la red del bosque de referencia sea más anidada, asimétrica y heterogénea, en relación con la encontrada en el bosque restaurado.

\section{MATERIALES Y MÉTODOS}

\section{Área de estudio}

El estudio se realizó en el parque ecológico La Poma, ubicado entre los 2600 y 2800 m de altitud en el municipio de Soacha, departamento de Cundinamarca, Colombia $\left(4^{\circ} 31^{\prime} 38.0^{\prime \prime}-74^{\circ} 16^{\prime} 55.2^{\prime \prime}\right)$. El parque cuenta con un área de 140.81 ha en la 
zona de vida de bosque seco montano según Holdridge (1966) y Riveros (2005). El parque presenta tres tipos de coberturas: la primera corresponde a un bosque andino secundario (42.51 ha); la segunda a los bosques de eucalipto (16.4 ha); y la tercera cobertura corresponde a zonas disturbadas (81.9 ha) (Salazar, 2003). En esta última en 40.39 ha se ha realizado el proceso de restauración ecológica desde 1996.

Durante la implementación del proyecto de restauración, en 1996, el bosque secundario nativo fue considerado como el sitio de referencia. Las principales familias de plantas son Sapindaceae, Boraginaceae y Primulaceae (Salazar, 2003), mientras que las especies de plantas más importantes son Miconia squamulosa (Melastomataceae), Cavendishia bracteata (Ericaceae) y Myrcianthes leucoxyla (Myrtaceae) (Salazar, 2003).

\section{Toma de datos}

\section{Muestreo de plantas en fruto}

En cada uno de los bosques se instalaron, de forma sistemática y aleatoria, cinco parcelas de $50 \times 20 \mathrm{~m}$, separadas por una distancia mínima de 200 m (Vázquez et al., 2009), para totalizar 1 ha por bosque. Durante 10 meses consecutivos en cada parcela se registraron y marcaron de forma mensual todas las plantas leñosas (arbustos y árboles) en fruto. Para cada individuo se calculó el tamaño de la cosecha a través de la metodología semicuantitativa de Fournier (1974). Así, el tamaño de la cosecha de cada individuo se valoró en una escala de 0 a 4 basado en el porcentaje de frutos en la copa del árbol; en donde 0 fue la ausencia de frutos, 1 correspondió a 1-25\% de la copa con fruto; 2: de 26-50\%; 3: de 51-75\%; y 4: de 76-100\%.

Las plantas en fruto registradas se consideraron ornitócoras si: I) por observación directa sus frutos fueron consumidos por algún ave; II) si por registros de literatura fueron reportadas en la dieta/ consumo de aves; o III) si los frutos presentaron características típicas para atraer dispersores tales como los frutos pequeños, esféricos, pulpa carnosa y colores como el negro, morado, naranja o rojo (Clout y Hay, 1989; Levey et al., 2005). Se colectaron especímenes de las plantas registradas en los censos y la identificación se realizó en el herbario forestal Gilberto Emilio Mahecha Vega UDBC, de la Universidad Distrital Francisco José de Caldas.

\section{Muestreo de aves frugívoras}

En las parcelas mencionadas se instalaron, al azar, cinco puntos de radio fijo de $25 \mathrm{~m}$ (Ralph, 1997). En estos puntos se realizaron mensualmente censos de aves entre las 6 y las 11 a. m., durante 20 minutos por punto de observación, para un total de 23 horas y 33 minutos por sitio (Ralph, 1997). Durante cada muestreo se anotaron todas las aves que pasaron dentro del radio de observación. La identificación de las aves se realizó a través de la guía de aves de Colombia (Hilty et al., 1986) y la guía de campo de las aves de la sabana de Bogotá (ABO et al., 2000). Las observaciones se realizaron durante 10 meses y no se hicieron registros durante horas de lluvia. Las aves se clasificaron como frugívoras si, por observación directa, consumieron frutos durante los censos y observaciones ad libitum, así como por información primaria, secundaria y literatura gris.

\section{Caracterización morfológica de frutos y aves}

Se tomó una muestra de mínimo 50 frutos para cada planta en fruto registrada en el área de estudio. Por cada fruto se midió el tamaño longitudinal y ecuatorial con un calibrador digital con exactitud $0.01 \mathrm{~mm}$. También se anotó el color y el tipo de fruto carnoso como baya, drupa, pepo y poma (Glimn-Lacy y Kaufman, 2006). Para el caso de las aves, basados en especímenes de museos, se realizaron mediciones a 10 pieles de ejemplares de machos y hembras por especie (Vazquéz, 2009). A cada ejemplar se le midió la comisura, el ancho del pico, la altura y la longitud del culmen total con un calibrador digital con exactitud $0.01 \mathrm{~mm}$ y se obtuvo un promedio para cada rasgo del pico por especie. Se consultaron ejemplares del Museo 
de Historia Natural de Instituto de Ciencias de la Universidad Nacional de Colombia y la colección de aves del Departamento de Biología de la Universidad Francisco José de Caldas.

\section{Análisis de datos}

Para los dos bosques se calculó la riqueza, la diversidad y el recambio de especies tanto de aves frugívoras como de plantas ornitócoras. Estas medidas se calcularon mediante el número de especies, el índice de diversidad de Shannon y de Jaccard, respectivamente. Además, con el fin de determinar si hubo diferencias significativas en la abundancia y riqueza de especies entre meses, se realizó un análisis de varianza simple para las aves y un análisis de varianza de medidas repetidas para las plantas (Durigan et al., 2015).

\section{Construcción de las redes}

Para cada bosque se realizó una red cuantitativa simulada para determinar la estructura de las interacciones. Para la construcción de las redes en cada bosque (referencia y restaurado) se tuvo en cuenta la coincidencia de rasgos y la abundancia relativa tanto de plantas como de aves. En primer lugar, para evaluar la coincidencia de rasgos se consideró lo propuesto por Gonzalez y Loiselle (2016), para quienes los desajustes morfológicos en la longitud de la comisura del pico del ave y el tamaño del fruto son los que limitan la ingestión de los frutos por parte de las aves. Así, si el límite inferior del tamaño de la comisura del pico es mayor que el límite inferior del tamaño del fruto la interacción es $100 \%$ posible con un valor de celda 1. Por el contrario, si el límite superior del rango del tamaño de la comisura es menor que el límite inferior del tamaño del fruto, la interacción es imposible y su valor de celda es 0 . En segundo lugar, con respecto a la abundancia relativa, para el caso de las aves se calculó la media por mes y por punto de muestreo (Ralph, 1996), mientras que para las especies en fruto esta se calculó usando el índice de Fournier (1974) por mes y por punto de muestreo.

En el caso de la red cuantitativa se simuló la frecuencia de interacción esperada de plantas y aves. Para el caso de las plantas se asumió que a mayor tamaño del fruto menor es la frecuencia de interacción (ecuación 1; González-Castro et al., 2015; Donoso et al., 2017).

$$
\mathrm{F}_{\mathrm{i}}=\frac{1}{x i}
$$

donde, Fi representa la frecuencia de interacción de la planta esperada y $\mathrm{x}_{\mathrm{i}}$ representa el tamaño de la semilla (en $\mathrm{mm}$ ) para la planta $i$.

Para el caso de las aves se asumió que, a pesar de que a mayor tamaño del pico la frecuencia de interacción disminuye, esta relación es menor debido a que las especies más grandes tienden a consumir más diversidad de frutos por individuo (ecuación 2, Donoso et al, 2017).

$$
\mathrm{g}_{\mathrm{i}}=\left(\frac{1}{y i}\right)+\beta
$$

donde, $\mathrm{g}_{\mathrm{i}}$ representa la frecuencia de interacción del ave esperada, $y_{i}$ representa el valor del tamaño del pico y $\beta$ es un parámetro de subcompensación, en el cual la frecuencia de interacción disminuye menos rápidamente cuan más grande es el tamaño del ave establecido en el $10 \%$ del valor máximo de 1/y (Donoso et al., 2017).

Posteriormente, se utilizó un modelo de nicho cuantitativo de Fründ et al. (2016) y Donoso et al. (2017) para calcular matrices de preferencias de las especies (ecuación 3).

$$
\mathrm{P}_{\mathrm{i}, \mathrm{j}}=\frac{e^{\left(-\left(\log (s(y j-x i))^{2} / 2\right.\right.}}{\sqrt{2 \pi\left(s(y i-x i)+e^{-1}\right)}}
$$

donde, $\mathrm{Pi}, \mathrm{j}$ es la preferencia de las especies de aves $j$ para ciertas especies de plantas $i ; y_{j}$ y $x_{i}$ son el tamaño de las aves y el tamaño de los frutos, respectivamente. El parámetro $s$ controla el grado de especialización de la interacción. Así, cuanto mayor sea la diferencia entre el tamaño del fruto y 
el tamaño del pico del ave, menor será la especialización. Por el contrario, cuanto menor sea esta diferencia, mayor será la especialización. Cuando no existe diferencia, es decir, cuando $y_{j}=x_{i}$ la coincidencia de los rasgos es perfecta $y$, por consiguiente, se considera la interacción como altamente especializada (Fründ et al., 2016).

\section{Análisis de las redes}

Se comparó la estructura de la red cuantitativa obtenida para el bosque restaurado y el de referencia. Esto se hizo utilizando métricas como el número medio de enlaces, la conectividad, el anidamiento, la diversidad de interacción, especialización y la asimetría de la red.

Primero, el número medio de enlaces representa el número medio de interacciones que establece una especie con otras $y$, en este sentido, permite reconocer las especies más conectadas o con mayor número de interacciones que se podrían considerar como especies claves en procesos de dispersión de semillas (Heleno et al., 2012).

Segundo, la conectividad se refiere a la proporción de interacciones realizadas del total de posibles entre las especies de una red. En ese sentido, una mayor conectividad indicaría una mayor complejidad en las interacciones que, según algunos autores, sería una ventaja adaptativa que protege a las comunidades de las extinciones secundarias (Heleno et al., 2012).

Tercero, el anidamiento es la tendencia de las especies especialistas de interactuar con especies generalistas (aquellas con mayor número de interacciones). Este se mide en una escala de 1 a 100 y brinda información acerca de la estabilidad y persistencia de la red (Nogales et al., 2015).

Cuarto, la diversidad de interacción es análoga a la diversidad de especies. Esta indica que a mayor diversidad de interacción hay mayor diversidad de respuesta físicas, fisiológicas o comportamentales frente a cambios ambientales (Snyder et al., 2006; Tylianakis et al., 2010).

Quinto, la especialización es la tendencia de una especie a preferir ciertas especies. Esto permite identificar especies que pueden llegar a ser más vulnerables frente a algún cambio en la red y se mide en un rango de 0 (sin especialización) a 1 (alta especialización) (Tylianakis et al., 2010). La asimetría de interacción muestra la igualdad o no de interacción y la dependencia de unas especies frente a otras (Tylianakis et al., 2010). Esta se midió en una escala de 0 (red simétrica) a 1 (red asimétrica).

Finalmente, para el análisis de las redes cualitativas se comparó el número medio de enlaces, el número medio de especies de la red y las variaciones mensuales para estos valores. Todos los análisis se hicieron con Past 3.21 y $R$ 6.3.0 con los paquetes Bipartite y Network y la función Aov para el análisis de varianza simple (Dormann et al., 2008; R Studio Team, 2015).

\section{RESULTADOS}

Se registraron 14 especies de aves frugívoras y 22 de plantas ornitócoras en fruto durante en un periodo de 10 meses consecutivos. Se encontró que la abundancia, riqueza y diversidad tanto de plantas ornitócoras como de aves frugívoras fueron mayores en el bosque de referencia que en el bosque restaurado (tabla 1). La abundancia de plantas ornitócoras fue significativamente mayor en el bosque de referencia (Anova de medidas repetidas $\mathrm{F}=3.255$ y $\mathrm{P}=0.0032$ ), mientras que las diferencias en la riqueza no fueron significativas $(\mathrm{F}=2.7$ y $\mathrm{P}=0.13)$. Para el caso de las aves la abundancia y la riqueza entre los dos bosques no presentaron diferencias significativas $(\mathrm{F}=1.503$ y $\mathrm{P}=$ $0.255 ; \mathrm{F}=2.7$ y $\mathrm{P}=0.139$, respectivamente) (tabla 1).

Se encontró una menor abundancia y riqueza en la oferta mensual de frutos en el bosque restaurado que en el bosque de referencia (figura 1). En los dos sitios el pico de fructificación se presentó en los meses de abril y octubre (Anova de medidas repetidas $\mathrm{F}=9$ y $\mathrm{P}=3.22 \times 10^{-11}$ ). Por otro lado, la abundancia mensual de aves frugívoras no presentó diferencias (Anova $\mathrm{F}=15.0$ y $\mathrm{F}=0.255$ ). La menor abundancia de aves coincidió con la menor oferta de frutos en los dos bosques (figura 1). 
Tabla 1. Abundancia, riqueza y diversidad de plantas ornitócoras en fruto y aves frugívoras entre marzo y diciembre de 2018

\begin{tabular}{ccccc}
\hline \multirow{2}{*}{ Indicador } & \multicolumn{2}{c}{ Aves frugívoras } & \multicolumn{2}{c}{ Plantas ornitócoras } \\
\cline { 2 - 5 } & Bosque referencia & Bosque restaurado & Bosque referencia & Bosque restaurado \\
\hline Abundancia & 152 & 93 & 162 & 93 \\
Riqueza & 13 & 10 & 18 & 16 \\
Índice Shannon_H & 2.03 & 1.75 & 2.50 & 2.22 \\
Índice Simpson_1-D & 0.83 & 0.78 & 0.89 & 0.84 \\
Equitatividad & 0.79 & 0.76 & 0.86 & 0.80 \\
Índice de Jaccard & 0.64 & 0.64 & 0.61 & 0.61 \\
\hline
\end{tabular}
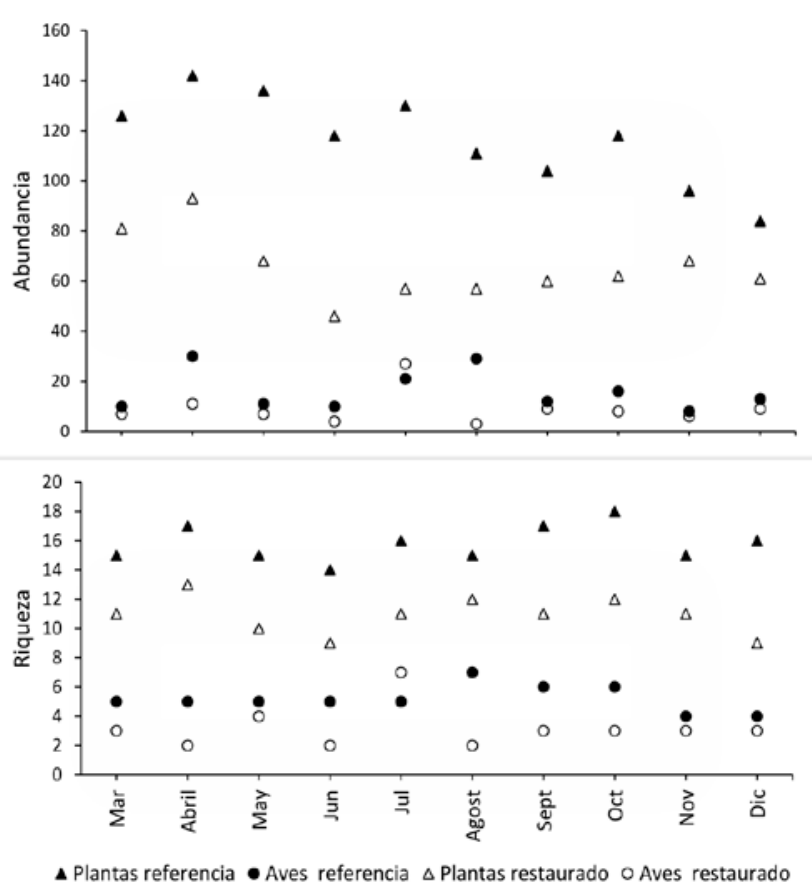

Figura 1. Abundancia y riqueza de aves frugívoras y plantas ornitócoras en fruto entre marzo y diciembre de 2018 en un bosque un bosque andino restaurado y el de referencia del parque ecológico La Poma, Cundinamarca.

\section{Redes mutualistas}

Al analizar las potenciales interacciones de dispersión en los bosques se encontró que la red cuantitativa del bosque de referencia presentó un mayor número de enlaces, un mayor anidamiento y asimetría; pero una conectividad similar al bosque restaurado (tabla 2). Tanto en el bosque restaurado como en el de referencia la especie más conectada y con un mayor número de interacciones fue el ave Diglossa cyanea (figuras 2 y 3). En el bosque restaurado Pipraeidea melanota presentó un gran número de interacciones mientras que $D$. cyanea presentó mayor número de enlaces en el bosque de referencia (figuras 2 y 3 ).

Tabla 2. Tipología de la red simulada de dispersión de semillas del bosque andino restaurado y el bosque de referencia

\begin{tabular}{ccc}
\hline Tipología de la red & $\begin{array}{c}\text { Bosque de } \\
\text { referencia }\end{array}$ & $\begin{array}{c}\text { Bosque } \\
\text { restaurado }\end{array}$ \\
\hline $\begin{array}{c}\text { Número de enlaces } \\
\text { por especie }\end{array}$ & 7.33 & 5.88 \\
Conectividad & 0.99 & 0.98 \\
Anidamiento & 13.0 & 23.3 \\
$\quad \begin{array}{c}\text { Diversidad de } \\
\text { interacción (Shannon) }\end{array}$ & 3.96 & 4.18 \\
Especialización & 0.17 & 0.14 \\
Asimetría & 0.13 & 0.20 \\
\hline
\end{tabular}

Adicionalmente, se encontró que en los dos bosques hay una baja tendencia de las especies especialistas a interactuar con especies generalistas (bajo anidamiento; tabla 2). No obstante, esta interacción es mayor en el bosque restaurado. De igual forma, la especialización fue muy baja en los dos sitios. Finalmente, las dos redes fueron simétricas, lo que implica que en los dos bosques la proporción de interacciones es similar entre las especies. 


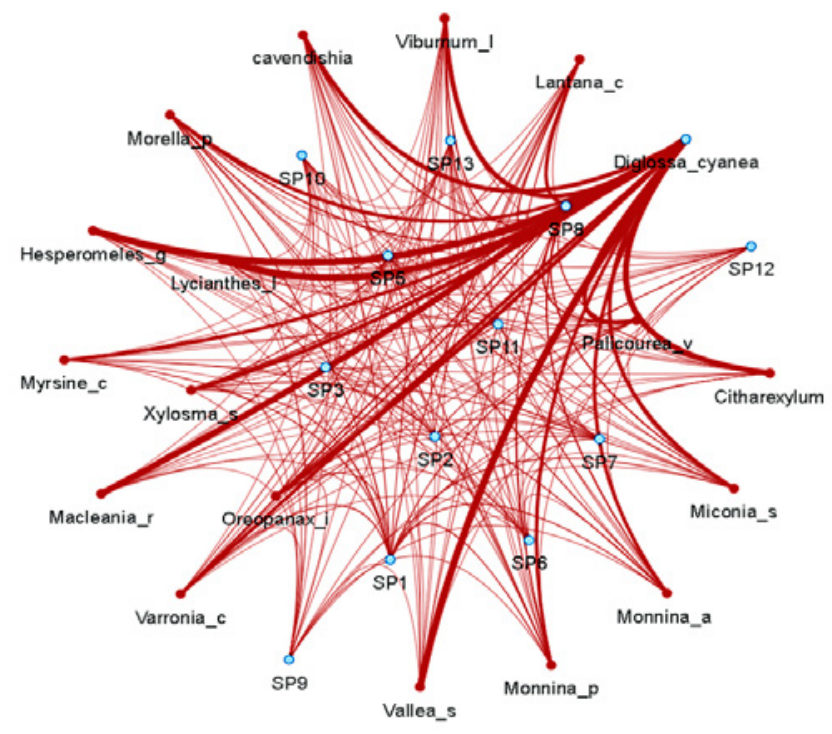

Figura 2. Frecuencia de interacción entre las plantas ornitócoras (círculo rojo) y las aves frugívoras (círculo azul claro) en la red del bosque de referencia, a partir de la abundancia (plantas y aves) y la coincidencia de rasgos (tamaño del fruto y la comisura de las aves); donde, a mayor grosor de la línea mayor frecuencia de interacción. Se muestra la especie Diglossa cyanea como la especie con mayor frecuencia de interacción con las plantas ornitócoras. Las demás especies de aves se representan con las abreviaturas: SP1 (Anisognathus igniventris), SP2 (Atlapetes pallidinucha), SP4 (Chlorornis riefferii), SP5 (Icterus chrysater), SP6 (Mimus gilvus), SP7 (Pheucticus aureoventris), SP8 (Patagioenas fasciata), SP9 (Tyrannus tyrannus), SP10 (Penelope montagnii), SP11 (Thraupis cyanocephala), SP12 (Turdus fuscater) y SP13 (Thraupis palmarum).

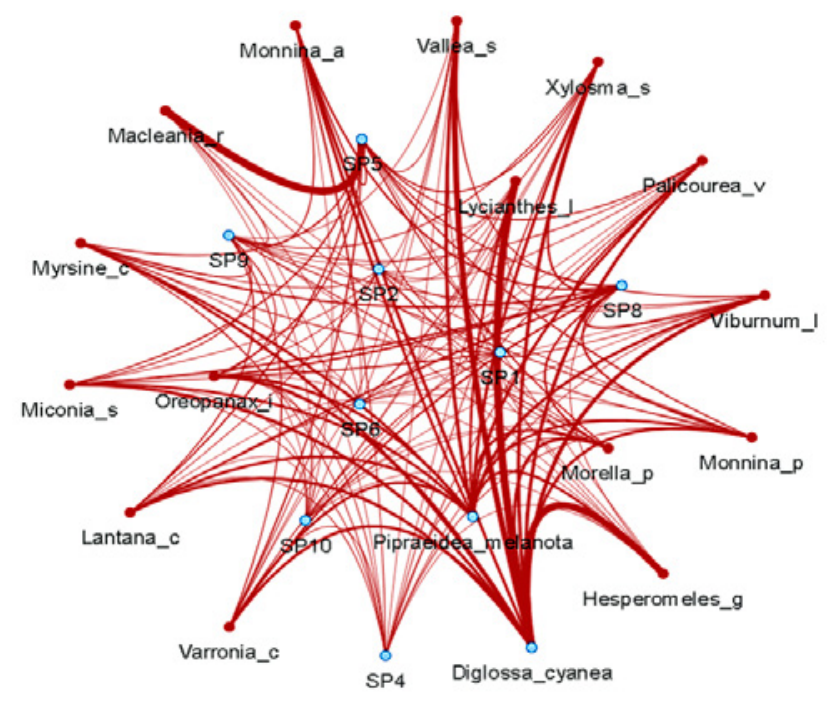

Figura 3. Frecuencia de interacción entre plantas ornitócoras (círculo rojo) y aves frugívoras (círculo azul claro) en la red del bosque restaurado a partir de la abundancia (plantas y aves) y la coincidencia de rasgos (tamaño del fruto y la comisura de las aves); donde, a mayor grosor de la línea mayor frecuencia de interacción. Se muestra la especie Diglossa cyanea (Sp3) y sp 7 (Pipraedea melonata). Las demás especies de aves se representan con las abreviaturas: SP1 (Anisognathus igniventris), SP2 (Atlapetes pallidinucha), SP4 (Icterus chrysater), SP5 (Patagioenas fasciata), SP6 (Pheucticus aureoventris), SP8 (Thraupis cyanocephala), SP9 (Thraupis palmarum) y SP10 (Turdus fuscater). 
Con respecto al número de interacciones por especie, entre un mes y otro variaron de forma; siendo mucho más marcada en el bosque restaurado que en el bosque de referencia (figura 4). Así, se registraron cambios de 1.6 enlaces por especie a 4.21 enlaces de un mes a otro en el bosque restaurado; mientras que en el bosque de referencia la diferencia entre un mes y otro no fue superior a 0.5 enlaces por especie. Esto indica una mayor estabilidad temporal de las interacciones en el bosque de referencia.

\section{DISCUSIÓN}

Las redes de interacción mutualista proveen información valiosa sobre los procesos ecológicos y las posibles funciones de las especies en los bosques (Bender et al., 2018). En este caso particular, el análisis de la red permitió evaluar el nivel de avance de la restauración en términos del restablecimiento de las potenciales relaciones de dispersión de semillas, lo cual no hubiera sido posible de evaluar únicamente la composición y la riqueza de algún grupo indicador (Ramírez, 2019). Así, los resultados presentados muestran que después de 22 años de un proceso de restauración de un potrero a un bosque andino seco, a diferencia de los datos de diversidad en donde hay diferencias significativas entre sitios, la red mutualista potencial de dispersión de semillas sí es diferente en ciertos aspectos entre el bosque restaurado y el bosque de referencia.

En primer lugar, el menor número de enlaces por especie en el bosque restaurado en relación al bosque de referencia refleja que, en 22 años, la recuperación de los procesos e interacciones a partir de actividades de restauración es lenta (Alarcón et al., 2008), a pesar de que la conectividad en los dos bosques presentó valores similares (tabla 2). Esto coincide con los resultados encontrados en un bosque de la Mata Atlántica brasilera restaurado hace 15 años, en el cual las interacciones de dispersión de semillas por aves fueron menores en comparación con bosques cuyo tiempo desde la restauración es mayor (Ribeiro da Silva et al., 2015). Posiblemente, el menor número de enlaces por especie en el bosque restaurado se debe a que la abundancia tanto de plantas como de aves fue menor que la encontrada en el bosque de referencia;

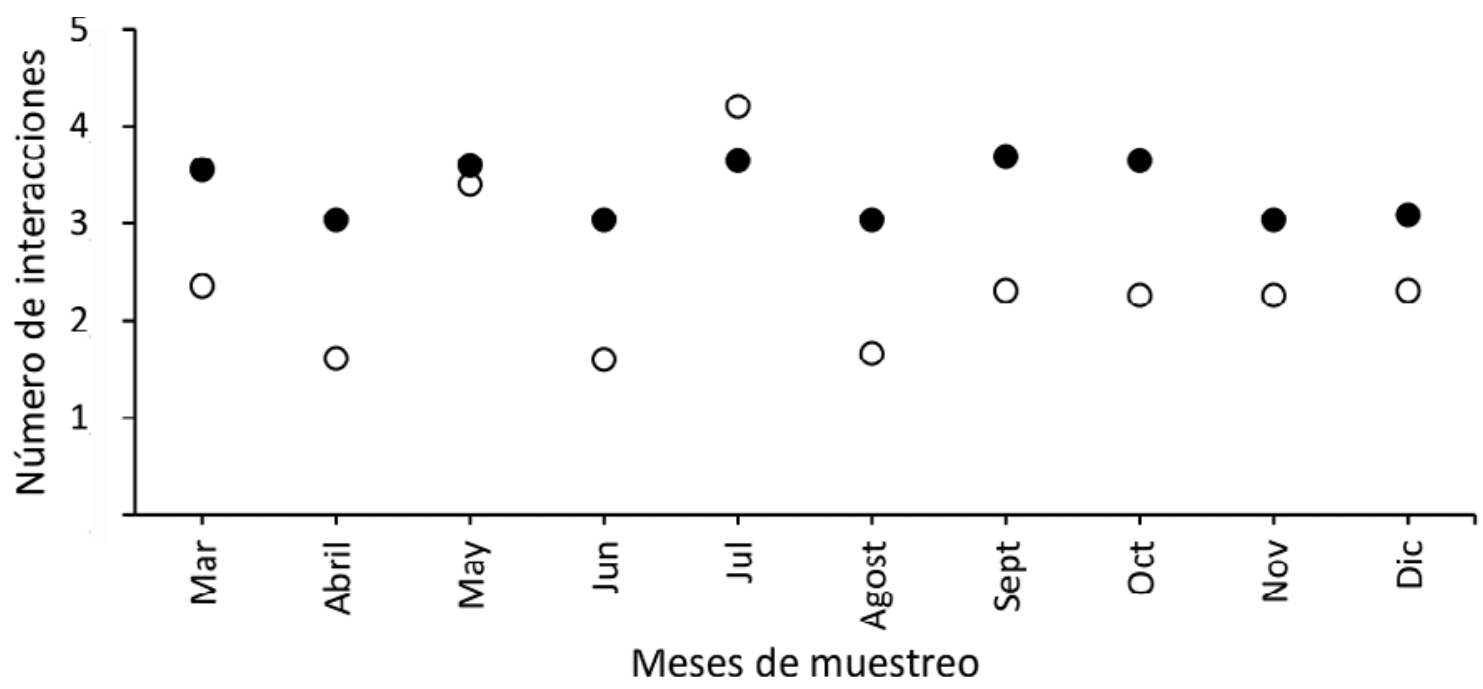

Figura 4. Número de interacciones mensuales de la red del bosque andino restaurado (circulo blanco) y de referencia (círculo negro), en el parque ecológico La Poma, Cundinamarca, donde se muestra en número de interacciones entre plantas ornitócoras y aves frugívoras en cada lugar. 
lo que implica que los procesos de dispersión son igualmente menores. Tal parece que una mayor cantidad de especies e individuos generan una mayor oportunidad de encontrar parejas de interacción (Alarcón et al., 2008; Olesen et al., 2008; Stang et al., 2007; Vázquez y Aizen, 2004). Estudios realizados con polinizadores e insectos en los que se ha encontrado una mayor cantidad de interacciones en hábitats con mayor riqueza de especies y abundancia de individuos soportan dicha situación (Alarcón et al., 2008; Banašek-Richter et al., 2004; Tylianakis et al., 2007). Así pues, la mayor abundancia y riqueza de plantas ornitócoras encontradas el bosque de referencia sugiere que hay una mayor oferta de frutos para las aves frugívoras y, por ende, un mayor número de enlaces en la red.

Lo anterior también coincide con las variaciones en el número de enlaces a lo largo del tiempo en el bosque restaurado, las cuales parecen indicar que la red es más inestable. Posiblemente, esto se debe a la estacionalidad en la oferta de frutos, pues en julio, por ejemplo, la principal fuente de frutos en el bosque restaurado es la uva camarona (Macleania rupestris) y del sauco de monte (Viburnum lasiophyllum); mientras que, en el bosque de referencia, hay una mayor diversidad en la oferta en los mismos meses como la uva de anís (Cavendishia bracteata), el raque (Vallea stipularis), palicourea (Palicourea vaginata), el sauco de monte (Viburnum lasiophylum) y el cucharo (Myrsine coriacea). Así, ante la menor oferta de frutos en el bosque restaurado las aves tienden a optar por recursos alternativos o cambiar entre hábitats (Carnicer et al., 2009), lo que podría incidir en un menor número de interacciones en el bosque restaurado.

En segundo lugar, la red del bosque restaurado presenta un menor anidamiento y asimetría de las interacciones en comparación con el bosque de referencia (tabla 2), lo que indica que las interacciones entre plantas ornitócoras y aves frugívoras tienden a ser homogéneas. Esto se debe, posiblemente, a la menor riqueza de plantas ornitócoras, así como a una menor abundancia en este bosque (tabla 1). Por ende, las interacciones tienden a ser las mismas entre las especies, ya que la diversidad de plantas no es alta. Estudios como los de Bascompte et al. $(2003 ; 2009)$ resaltan la importancia de una alta asimetría de la red, pues estas ayudan al mantenimiento de la biodiversidad y a que haya una baja dependencia de las interacciones entre las especies. En este sentido, una baja riqueza y abundancia de plantas y aves en este estudio coincide con una baja asimetría de las interacciones en el bosque restaurado.

Además, varios estudios de redes mutualistas muestran que un bajo anidamiento y asimetría de la red son indicadores de que estas interacciones tienden a ser más susceptibles frente a perturbaciones y cambios ambientales (Bascompte et al., 2009; Vazquéz et al., 2009; Tylianakis 2010). Por lo tanto, las interacciones de la red del bosque restaurado pueden ser más inestable que las del bosque de referencia, ya que, frente a la pérdida de una especie de planta o ave, podrían afectar a las demás especies que interactúan en la red.

Al contrastar nuestros resultados con otras redes en zonas andinas se encuentra que tanto el bosque restaurado como el bosque de referencia aquí estudiado tienen un bajo anidamiento (pocas interacciones de especies especialistas con las especies más conectadas), una alta simetría (interacciones similares entre especies) y una baja especialización en relación a dichos estudios. Por ejemplo, Palacio et al. (2016) encontraron que en un bosque andino conservado del Valle del Cauca la red de dispersión fue muy anidada, asimétrica y modular. De igual forma, en bosques conservados en diferentes partes del mundo se han encontrado patrones similares (Bascompte, 2009; Bascompte et al., 2003; González-Castro et al., 2015; Nogales et al., 2015). En este sentido, los valores bajos de anidamiento y especialización tanto en el bosque restaurado como el de referencia, así como una alta simetría de las redes, podrían indicar que estos bosques se encuentran en un estado temprano/ 
intermedio de sucesión. En este sentido, tal parece que el bosque de referencia a pesar de llevar más de 70 años sin intervención (Salazar, 2003) no es un bosque bien conservado, sino que aún necesita muchos años para su recuperación. Esto coincide con Groenendijk (2005), quien sostiene que la recuperación de los bosques andinos secos es muy difícil, incluso bajo procesos de restauración asistida. Adicionalmente, este bajo anidamiento y baja asimetría pueden ser producto de la ausencia de ciertas especies clave tanto de plantas como de animales que estimularían la ocurrencia de ciertas interacciones.

A pesar de esta posible ausencia de especies claves tanto de plantas como de aves, se encontraron algunas especies altamente conectadas que desempeñarían un papel importante en procesos de dispersión. Tal es el caso del ave Diglossa cyanea, la cual presentó una alta frecuencia de interacción con varias especies de plantas ornitócoras (figuras 3 y 4) debido a una mayor coincidencia de rasgos morfológicos del tamaño del fruto y el pico. En el caso de las plantas, la uva camarona (Macleania rupestris), el raque (Vallea stipularis) y la uva de anís (Cavendishia bracteata) también se podrían considerar especies clave, ya que estuvieron altamente conectadas y fueron parte importante de la oferta de frutos durante los meses de muestreo. Estas plantas presentan bayas rojas y carnosas, lo que las haría altamente atractivas para aves dispersoras. Por otro lado, es de resaltar que las aves generalistas Patagioenas fasciata y Turdus fuscater fueron más importantes en el bosque de referencia que en el restaurado. Estudios como los de Cusser y GoodeIl (2013) y González et al. (2010) sugieren que las especies generalistas son valiosas ya que mantienen la conectividad y estabilidad de la red, pues en últimas terminan transportando semillas de una gran cantidad de especies ornitócoras.

Por último, a pesar de que probablemente evaluación de la trayectoria de la restauración a través de redes subestima algunas interacciones reales (debido a que no se registró la frecuencia del consumo de los frutos por parte de las aves), creemos que es una importante aproximación al monitoreo de la recuperación (o no) de las interacciones después de un proceso de restauración. Esto se debe a que al evaluar la diversidad tanto de plantas como de aves en los dos bosques no se encontraron diferencias significativas (tabla 1), lo que indicaría que la trayectoria de la restauración es positiva. Sin embargo, al evaluar mediante la red mutualista sí se encontraron diferencias importantes entre bosques y, además, da luces sobre cuáles especies pueden ser consideradas como claves y ser manejadas para acelerar, aún más, los procesos de restauración.

En ese sentido, procesos de enriquecimiento del bosque restaurado con especies vegetales como Cavendishia bracteata, Miconia squamulosa y Palicourea vaginata, así como el incremento de las abundancias de Vallea stipularis, Myrsine coriacea y Hesperomeles goudotiana, podrían mejorar las interacciones no solo con las aves, sino también con mamíferos. Ello repercutiría en una mayor dispersión y lluvia de semillas y, por ende, en la aceleración de la restauración. También se podría considerar enriquecer el bosque con plantas con variados tipos y tamaños de frutos, cuyas fenologías sean diferentes para así lograr, por un lado, atraer una mayor diversidad y variedad de dispersores y, por el otro, lograr una mayor variabilidad de oferta de frutos durante el año y así, enriquecer de esta manera el banco de semillas en el suelo.

\section{CONCLUSIONES}

El análisis de redes presentado en este artículo muestra información adicional que no hubiera sido posible obtener al evaluar la composición de especies indicadoras como las aves. Las interacciones entre plantas ornitócoras y aves frugívoras potenciales nos permitieron valuar que, aunque la trayectoria de la restauración tiende a semejarse al bosque de referencia, aún le falta camino por 
recorrer en el restablecimiento de las interacciones. En el mismo sentido, fue posible identificar especies claves tanto de plantas como de aves que desempeñan funciones importantes en los procesos de dispersión de semillas y aquellas que pueden ser utilizadas en la aceleración de la restauración. Por ejemplo, se identificaron especies como el ave Diglossa cyanea, la cual presentó una gran conectividad con otras especies de plantas, lo que sugiere un papel importante en la dispersión de semillas de los bosques andinos.

Finalmente, teniendo en cuenta que el bosque de referencia presentó un bajo anidamiento a pesar de llevar más de 70 años bajo protección, se sugiere trabajar en el enriquecimiento de este bosque y la recuperación de su integridad. Para este fin, futuros estudios deberían comparar el estado de las redes de interacción de este bosque en relación con un bosque más conservado para guiar dichos procesos de enriquecimiento. De igual forma, sería interesante contrastar la red simulada a partir de rasgos morfológicos con una red que incluya la frecuencia del consumo real de las aves frugívoras para conocer el nivel de error y subestimación que conlleva la construcción de redes a partir de rasgos. Por último, se recomienda realizar monitoreo y seguimiento a otros aspectos funcionales asociados a redes mutualistas como la polinización y la herbivoría entre los dos lugares evaluados.

\section{AGRADECIMIENTOS}

Las autoras agradecen a la Corporación Ambiental Empresarial, administradores del parque ecológico La Poma por el apoyo al trabajo de campo. De igual forma, agradecemos a Rocío Cortés, Oscar Laverde y a Marcia Muñoz por sus valiosos comentarios a versiones previas a este manuscrito.

\section{CONFLICTO DE INTERESES}

Las autoras declaran no tener conflicto de intereses.

\section{CONTRIBUCIÓN POR AUTOR}

Ambas autoras idearon la investigación. A.R. realizó la toma de datos y el análisis de las redes. A.R. y A.P.R. redactaron el manuscrito. A.R. y A.P.R. contribuyeron a la discusión y comentaron los borradores previos.

\section{REFERENCIAS}

ABO, A. B., Stiles, F. G., Bohórquez, C. I. y Cadena, C. D. (2000). Aves de la sabana de Bogotá: Guía de campo. Bogotá: ABO, CAR.

Alanís-Rodríguez, E., Jiménez-Pérez, J., Espinoza-Vizcarra, D., Jurado-Ybarra, E., Aguirre-Calderón, O. A. y González-Tagle, M. A. (2008). Evaluación del estrato arbóreo en un área restaurada post-incendio en el parque ecológico Chipinque, México. Revista Chapingo Serie Ciencias Forestales y del Ambiente, 14(2), 113-118. https://doi.org/10.5154/r.rchscfa.2010.05.032

Alarcón, R., Waser, N. M. y Ollerton, J. (2008). Year-toyear variation in the topology of a plant-pollinator interaction network. Oikos, 117(12), 1796-1807. https://doi.org/10.1111/j.0030-1299.2008.16987.x

Arbeláez, M. V. y Parrado-Rosselli, A. (2005). Seed dispersal modes of the sandstone plateau vegetation of the middle Caquetá river region, Colombian Amazonia. Biotropica, 37(1), 64-72. https://doi.org/10.1111/j.1744-7429.2005.03077.x

Banašek-Richter, C., Cattin, M. F. y Bersier, L. F. (2004). Sampling effects and the robustness of quantitative and qualitative food-web descriptors. Journal of Theoretical Biology, 226(1), 23-32. https://doi.org/10.1016/s0022-5193(03)00305-9

Bascompte, J. (2009). Mutualistic networks. Frontiers in Ecology and the Environment, 7(8), 429-436. https://doi.org/10.1890/080026

Bascompte, J., Jordano, P., Melián, C. J. y Olesen, J. M. (2003). The nested assembly of plant-animal mutualistic networks. Proceedings of the National Academy of Sciences, 100(16), 9383-9387. https://doi.org/10.1073/pnas.1633576100 
Bender, I. M., Kissling, W. D., Blendinger, P. G., Böhning-Gaese, K., Hensen, I., Kühn, I., Quitián, M. (2018). Morphological trait matching shapes plant-frugivore networks across the Andes. Ecography, 41(11), 1910-1919. https://doi.org/10.1111/ecog.03396

Burgos,J.,Villota,A.yFernández,S.J.T.(2003).Aplicación de la metodología de complejos simpliciales en las interacciones biológicas de Frugivoría y dispersión de semillas en grupo de aves de la Reserva Biológica de Carpanta. Colombia Forestal, 8(16), 32-47. https://doi.org/10.24201/eg.v5i0.358

Carnicer, J., Jordano, P. y Melián, C. J. (2009). The temporal dynamics of resource use by frugivorous birds: A network approach. Ecology, 90(7), 1958-1970. https://doi.org/10.1890/07-1939.1

Chazdon, R. L., Broadbent, E. N., Rozendaal, D. M., Bongers, F., Zambrano, A. M. A., Aide, T. M., Balvanera, P., Becknell, J. M., Boukili, V., Brancalion, P., Craven, D., Almeida-Cortez, J., Cabral, G., de Jong, B, Denslow, J., Dent, D., DeWalt, S., Dupuy, J., Durán, S., Espírito-Santo, M., Fandino, M., César, R., Hall J., Hernández-Stefanoni, J., Jakovac, C. [...] y Poorter, L. (2016). Carbon sequestration potential of second-growth forest regeneration in the Latin American tropics. Science Advances, 2(5), e1501639. https://doi.org/10.1126/sciadv.1501639

Clout, M. N. y Hay, J. R. (1989). The importance of birds as browsers, pollinators and seed dispersers in New Zealand forests. New Zealand Journal of Ecology, 12(supplement), 27-33. https://www.jstor.org/stable/24053245

Cusser, S. y Goodell, K. (2013). Diversity and distribution of floral resources influence the restoration of plant-pollinator networks on a reclaimed strip mine. Restoration Ecology, 21(6), 713-721. https://doi.org/10.1111/rec.12003

Dehling, D. M., Jordano, P., Schaefer, H. M., Böhning-Gaese, K. y Schleuning, M. (2016). Morphology predicts species' functional roles and their degree of specialization in plantfrugivore interactions. Proceedings of the
Royal Society B: Biological Sciences, 283, 20152444. https://doi.org/10.1098/rspb.2015.2444

Donoso, I., Schleuning, M., García, D. y Fründ, J. (2017). Defaunation effects on plant recruitment depend on size matching and size trade-offs in seed-dispersal networks. Proceedings of the Royal Society B: Biological Sciences, 284(1855), 20162664. https://doi.org/10.1098/rspb.2016.2664

Dormann, C. F., Gruber, B. y Fruend, J. (2008). Introducing the bipartite Package: Analysing Ecological Networks. R News, 8(2), 8-11.

Durigan, G., Domínguez-Haydar, Y., Brancalion, P., Aronson, J., Pizano, C. y Aguilar-Garavito, M. (2015). Monitoreo a procesos de restauración ecológica aplicado a ecosistemas terrestres. Instituto de Investigación de Recursos Biológicos Alexander von Humboldt.

Fontaine, C., Dajoz, I., Meriguet, J. y Loreau, M. (2005). Functional diversity of plant-pollinator interaction webs enhances the persistence of plant communities. PLoS Biol, 4(1), e1. https://doi.org/10.1371/journal.pbio.0040001

Forup, M. L., Henson, K. S., Craze, P. G. y Memmott, J. (2008). The restoration of ecological interactions: Plant-pollinator networks on ancient and restored heathlands. Journal of Applied Ecology, 45(3), 742-752. https://doi.org/10.1111/j.1365-2664.2007.01390.x

Fournier, L. (1974). Un método cuantitativo para la medición de características fenológicas en árboles. Turrialba, 24(4), 422-423.

Fründ, J., McCann, K. S. y Williams, N. M. (2016). Sampling bias is a challenge for quantifying specialization and network structure: Lessons from a quantitative niche model. Oikos, 125(4), 502-513. https://doi.org/10.1111/oik.02256

García, D. (2016). Birds in ecological networks: Insights from bird-plant mutualistic interactions. Ardeola, 63(1), 151-180. https://doi.org/10.13157/arla.63.1.2016.rp7

Glimn-Lacy, J. y Kaufman, P. B. (2006). Botany illustrated: Introduction to plants, major groups, flowering plant families. Springer Science \& Business Media. https://doi.org/10.2307/4448160 
González, A. M. M., Dalsgaard, B. y Olesen, J. M. (2010). Centrality measures and the importance of generalist species in pollination networks. Ecological Complexity, 7(1), 36-43. https://doi.org/10.1016/j.ecocom.2009.03.008

González-Castro, A., Yang, S., Nogales, M. y Carlo, T. A. (2015). Relative importance of phenotypic trait matching and species' abundances in determining plant-avian seed dispersal interactions in a small insular community. AoB Plants, 7, plv017. https://doi.org/10.1093/aobpla/plv017

Gonzalez, O. y Loiselle, B. A. (2016). Species interactions in an Andean bird-flowering plant network: phenology is more important than abundance or morphology. PeerJ, 4:e2789 https://doi.org/10.7717/peerj.2789

Groenendijk, J. P. (2005). Towards recovery of native dry forest in the Colombian Andes: $A$ plantation experiment for ecological restoration. Universiteit van Amsterdam, IBED. https://hdl.handle.net/11245/1.241741

Heleno, R., Devoto, M. y Pocock, M. (2012). Connectance of species interaction networks and conservation value: Is it any good to be well connected? Ecological Indicators, 14(1), 7-10. https://doi.org/10.1016/j.ecolind.2011.06.032

Hilty, S. L., Brown, W. L. y Brown, B. (1986). A guide to the birds of Colombia. University Press.

Holdridge, L. (1987). Ecología basada en las zonas de vida. Colección libros y materiales educativos; $\mathrm{n}$. 83.21 6 P. Instituto Interamericano de Cooperación para la Agricultura.

Howe, H. F. y Smallwood, J. (1982). Ecology of seed dispersal. Annual Review of Ecology and Systematics, 13(1), 201-228. https://doi.org/10.1146/annurev.es.13.110182.001221

Kaiser-Bunbury, C. N., Traveset, A. y Hansen, D. M. (2010). Conservation and restoration of plant-animal mutualisms on oceanic islands. Perspectives in Plant Ecology, Evolution and Systematics, 12(2), 131-143. https://doi.org/10.1016/j.ppees.2009.10.002

Kelly, D., Ladley, J. J., Robertson, A. W., Anderson, S. H., Wotton, D. M. y Wiser, S. K. (2010). Mutualisms with the wreckage of an avifauna: The status of bird pollination and fruit-dispersal in New Zealand. New Zealand Journal of Ecology, 34(1), 66. https://newzealandecology.org/nzje/2918

Levey, D. J., Bolker, B. M., Tewksbury, J. J., Sargent, S. y Haddad, N. M. (2005). Effects of landscape corridors on seed dispersal by birds. Science, 309(5731), 146-148. https://doi.org/10.1126/science.1111479

Link, A. y Stevenson, P.R. (2004). Fruit dispersal syndromes in animal disseminated plants at Tinigua National Park, Colombia. Revista Chilena de Historia Natural, 77(2), 319-334. https://doi.org/10.4067/s0716-078x2004000200010

Murcia, C. y Guariguata, M. R. (2014). La restauración ecológica en Colombia: tendencias, necesidades y oportunidades. Center for International Forestry Research.

Nogales, M., Heleno, R., Rumeu, B., González-Castro, A., Traveset, A., Vargas, P. y Olesen, J. M. (2015). Seed-dispersal networks on the Canaries and the Galápagos archipelagos: interaction modules as biogeographical entities. Global Ecology and Biogeography. https://doi.org/10.1111/geb.12315

Olesen, J. M., Bascompte, J., Elberling, H. y Jordano, P. (2008). Temporal dynamics in a pollination network. Ecology, 89(6), 1573-1582. https://doi.org/10.1890/07-0451.1

Palacio, R. D., Valderrama-Ardila, C. y Kattan, G. H. (2016). Generalist species have a central role in a highly diverse plant-frugivore network. Biotropica, 48(3), 349-355. https://doi.org/10.1111/btp.12290

Ralph, C. J., Geupel, G. R., Pyle, P., Martin, T. E., DeSante, D. F. y Milá, B. (1996). Manual de métodos de campo para el monitoreo de aves terrestres. https://doi.org/10.2737/psw-gtr-159

Ramírez, A. (2019). Evaluación de la trayectoria de la restauración a través de las aves: Análisis de la recuperación de la composición y las interacciones en un bosque andino (tesis de maestría). Universidad Distrital Francisco José de Caldas.

Ribeiro da Silva, F., Montoya, D., Furtado, R., Memmott, J., Pizo, M. A. y Rodrigues, R. R. (2015). 
The restoration of tropical seed dispersal networks. Restoration Ecology, 23(6), 852-860. https://doi.org/10.1111/rec.12244

Riveros, D. (2005). Aporte al conocimiento de la biodiversidad florística y faunística, manejo tecnológico de germoplasma y regeneración natural de especies nativas en el parque ecológico La Poma (trabajo de grado, Ingeniería Forestal). Universidad del Tolima, Bogotá, Colombia.

R Studio Team (2015) RStudio: Integrated Development for R. RStudio, Inc. (Computer Software v0.98.1074). http://www.rstudio.com/

Salazar, L. (2003). Aproximación a la diversidad florística y análisis sucesional de las coberturas vegetales para el periodo de 1943 a 2002 en el parque ecológico La poma. (trabajo de grado, Ingeniería Forestal). Universidad del Tolima, Bogotá, Colombia.

Santamaría, L. y Rodríguez-Gironés, M. A. (2007). Linkage rules for plant-pollinator networks: Trait complementarity or exploitation barriers? PLoS Biology, 5(2), e31. https://doi.org/10.1371/journal.pbio.0050031

Snyder, W. E., Snyder, G. B., Finke, D. L. y Straub, C. S. (2006). Predator biodiversity strengthens herbivore suppression. Ecology Letters, 9(7), 789-796. https://doi.org/10.1111/j.1461-0248.2006.00922.x

Stang, M., Klinkhamer, P. G. y Van der Meijden, E. (2007). Asymmetric specialization and extinction risk in plant-flower visitor webs: A matter of morphology or abundance? Oecologia, 151(3), 442-453. https://doi.org/10.1007/s00442-006-0585-y

Tylianakis, J. M., Laliberté, E., Nielsen, A. y Bascompte, J. (2010). Conservation of species interaction networks. BiologicalConservation, 143(10), 2270-2279. https://doi.org/10.1016/j.biocon.2009.12.004
Tylianakis, J. M., Tscharntke, T. y Lewis, O. T. (2007). Habitat modification alters the structure of tropical host-parasitoid food webs. Nature, 445(7124), 202. https://doi.org/10.1038/nature05429

Vázquez, D. P. y Aizen, M. A. (2004). Asymmetric specialization: A pervasive feature of plant-pollinator interactions. Ecology, 85(5), 1251-1257. https://doi.org/10.1890/08-1837.1

Vázquez, D. P., Chacoff, N. P. y CagnoIo, L. (2009). Evaluating multiple determinants of the structure of plant-animal mutualistic networks. Ecology, 90(8), 2039-2046. https://doi.org/10.1111/j.2007.0030-1299.15828.x

Vázquez, D. P., Melián, C. J., Williams, N. M., Blüthgen, N., Krasnov, B. R. y Poulin, R. (2007). Species abundance and asymmetric interaction strength in ecological networks. Oikos, 116(7), 1120-1127. https://doi.org/10.1111/j.2007.0030-1299.15828.x

Williams, N. M. (2011). Restoration of nontarget species: Bee communities and pollination function in riparian forests. Restoration Ecology, 19(4), 450-459. https://doi.org/10.1111/j.1526-100x.2010.00707.x

Wunderle, J. M. (1997). The role of animal seed dispersal in accelerating native forest regeneration on degraded tropical lands. Forest Ecology and Management, 99(1), 223-235. https://doi.org/10.1016/s0378-1127(97)00208-9

Yockteng, R. y Cavelier, J. (1998). Diversidad y mecanismos de dispersión de árboles de la Isla Gorgona y de los bosques húmedos tropicales del Pacífico colombo-ecuatoriano. Revista de Biología Tropical, 45-53. https://doi.org/10.15517/rbt.v67i6.37517

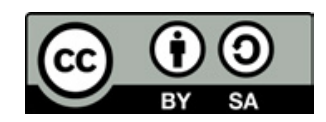

Colombia Forestal •ISSN 0120-0739• e-ISSN 2256-201X • Bogotá-Colombia • Vol. 24 No. 1 • Enero-Junio de $2021 ・$ pp. 108-122 [ 122 ] 